\title{
Comparison of the Transient Stress-Strain Response of Rubber to Its Linear Dynamic Behavior
}

\author{
Peter H. Mott, ${ }^{1}$ Jeffrey N. Twigg, ${ }^{1}$ C. Michael Roland, ${ }^{1}$ Kenneth E. Nugent, ${ }^{2}$ \\ Terry E. Hogan, ${ }^{3}$ Christopher G. Robertson ${ }^{3}$ \\ ${ }^{1}$ Chemistry Division, Naval Research Laboratory, Washington, District of Columbia 20375-5342 \\ ${ }^{2}$ Science Applications International Corporation, La Plata, Maryland 20646 \\ ${ }^{3}$ Bridgestone Americas, Center for Research and Technology, Akron, Ohio 44317-0001 \\ Correspondence to: P. H. Mott (E-mail: peter.mott@nrl.navy.mil)
}

Received 15 March 2011; revised 10 May 2011; accepted 17 May 2011; published online 20 June 2011

DOI: $10.1002 /$ polb.22292

ABSTRACT: Master curves of the small strain and dynamic shear modulus are compared with the transient mechanical response of rubbers stretched at ambient temperature over a seven-decade range of strain rates $\left(10^{-4}\right.$ to $\left.10^{3} \mathrm{~s}^{-1}\right)$. The experiments were carried out on 1,4- and 1,2-polybutadienes and a styrene-butadiene copolymer. These rubbers have respective glass transition temperatures, $T_{\mathrm{g}}$, equal to $-93.0,0.5$, and $4.1{ }^{\circ} \mathrm{C}$, so that the room temperature measurements probed the rubbery plateau, the glass transition zone, and the onset of the glassy state. For the 1,4-polybutadiene, in accord with previous results, strain and strain rate effects were decoupled (additive). For the other two materials, encroachment of the segmental dynamics precluded separation of the effects of strain and rate. These results show that for rubbery polymers near $T_{\mathrm{g}}$ the use of linear dynamic data to predict stresses, strain energies, and other mechanical properties at higher strain rates entails large error. For example, the strain rate associated with an upturn in the modulus due to onset of the glass transition was three orders of magnitude higher for large tensile strains than for linear oscillatory shear strains. (C) 2011 Wiley Periodicals, Inc.* J Polym Sci Part B: Polym Phys 49: 1195-1202, 2011

KEYWORDS: high strain rate; linear dynamic; mechanical properties; stress-strain behavior
INTRODUCTION Applications as diverse as impact protection, ${ }^{1}$ rubber catapults, ${ }^{2}$ skidding tires, ${ }^{3}$ and possibly even atherosclerotic blood flow ${ }^{4}$ subject elastomers to both large strains and high strain rates. ${ }^{5,6}$ However, material characterizations are usually limited to only one of these conditionsslow straining to the point of failure or rapid displacements of small amplitude. Consequently, the mechanical response for many applications is often predicted by extrapolating linear dynamic data, with the assumption that strain and rate effects are separable, ${ }^{7-14}$ and that the time-superposition principle is valid. However, at sufficiently high rates significant contributions to the mechanical response arise from the segmental dynamics, which are omitted if data used for extrapolations is limited to the rubbery behavior. ${ }^{1,15,16}$

Using small strain dynamic measurements to predict the stress-strain properties at higher rates and higher strains is analogous to comparing dynamic and steady shear flow viscosities using the empirical rule of Cox and Merz: ${ }^{17}$

$$
\left|\eta^{*}(\omega)\right|_{\dot{\gamma}=\omega}=\eta_{\mathrm{SS}}(\dot{\gamma}),
$$

where $\left|\eta^{*}\right|$ is the magnitude of the complex dynamic shear viscosity $\left(=\left|G^{*}\right| / \omega\right.$ with $\left|G^{*}\right|$ the magnitude of the complex shear modulus), $\eta_{\text {SS }}$ the steady-state viscosity measured in a flow experiment, $\omega$ the oscillation frequency, and $\dot{\gamma}$ the steady-state shear rate. This connection between the dynamic and steady-state response works reasonably well for linear polymers, ${ }^{18-21}$ and some branched polymers. ${ }^{22,23}$ Equation 1 is applicable to polymeric melts, because for crosslinked materials there is no steady-state shearing, only mechanical equilibrium after relaxation is complete.

Separability of time and strain allows deconvolution of the viscoelastic contributions from the stress through application of the Boltzmann superposition principle: ${ }^{24-27}$

$$
\sigma(t)=\int_{-\infty}^{t} E(t-\tau) \frac{\mathrm{d} \varepsilon(\tau)}{\mathrm{d} \tau} \mathrm{d} \tau,
$$

where $\varepsilon(t)$ is the strain as a function of time, and $E(t-t)$ is the linear (strain-independent) relaxation modulus. Equation 2 has been used to determine the form of the relaxation function consistent with the Cox-Merz rule. ${ }^{18,28}$ A modification allows separation of the stress response from the time response for higher strains ${ }^{25,29}$

$$
\sigma(t)=\int_{-\infty}^{t} E(t-\tau) g(\varepsilon) \frac{\mathrm{d} \varepsilon(\tau)}{\mathrm{d} \tau} \mathrm{d} \tau
$$


where the damping function $g(\varepsilon)$ represents the nonlinear, equilibrium or elastic behavior. For polyisoprene and styrene-butadiene rubbers, time and strain effects have been shown to be independent, with eq 3 yielding good agreement with experimental stress-strain curves taken to extensions as high as two; ${ }^{13}$ however, this approach fails when the sign of the strain changes, for example, for retraction of stretched rubber. ${ }^{13,30}$

Recent models have combined Prony series fits of linear viscoelastic data with quasistatic or equilibrium stress-strain data, to predict the mechanical response of a polymer at high strains and high strain rates. ${ }^{8,11,14,31,32}$ This approach simplifies the analysis, in comparison to other large strain constitutive models. ${ }^{33-35}$ The implication of the simpler approach is that an analog of the Cox-Mertz rule (eq 1) might be used to predict the sensitivity of stress to strain rate:

$$
\frac{\mathrm{d} \ln \sigma(\varepsilon, t)}{\mathrm{d} \ln \dot{\gamma}}=\frac{\mathrm{d} \ln \sigma^{*}}{\mathrm{~d} \ln \omega},
$$

where $\sigma(\varepsilon, t)$ is the stress as a function of strain $\varepsilon$ and time $t$ from the transient stress-strain curve, and $\sigma^{*}$ is the stress $\left(=\varepsilon\left|E^{*}\right|=3 \varepsilon\left|G^{*}\right|\right.$, where $\left|E^{*}\right|$ and $\left|G^{*}\right|$ are the respective complex Young's and shear modulus magnitude, respectively) found from the linear viscoelastic data, at some arbitrary strain $\varepsilon$. This selected strain shifts the $\sigma^{*}$ vertically and has no effect on the slope. This relation suggests similarities between steady-state shear of a melt and large elastic strains in rubber, although clearly there are significant differences as noted above.

The question is whether eq 4 makes accurate predictions for deformations at rates sufficient to involve the local segmental dynamics, which govern the onset of the glass transition relaxation. The segmental dynamics have quite different properties than the global chain motions probed at longer times and higher temperatures. ${ }^{5,16,36}$ To address this issue, we obtained stress-strain data at ambient temperature for three rubbers, networks of 1,2- and 1,4-polybutadienes (1,2$\mathrm{PB}$ and 1,4-PB, respectively) and a linear styrene-butadiene copolymer (SBR), using a novel instrument to access large strains over a wide range of strain rates. At the highest rates encroachment of the local segmental dynamics becomes apparent, to a degree dependent on the polymer $T_{\mathrm{g}}$.

\section{EXPERIMENTAL}

SBR was polymerized using butyl lithium as the initiator, with the polymer having $64.2 \%$ total styrene $\left({ }^{1} \mathrm{H}-\mathrm{NMR}\right)$, $35.8 \%$ present in blocks of three or more repeat units, and $35.8 \%$ butadiene that was $47.4 \%$ vinyl (1,2-addition product). The same initiator was used along with a polar modifier to promote 1,2-addition to synthesize primarily polyvinylethylene (1,2-PB), with $6.8 \%$ cyclic vinyl and 5.7\% 1,4content. The 1,4-PB was a commercial material, Taktene 550 from Lanxess, having 51\% trans-1,4-, 38\% cis-1,4-, and 11\% vinyl repeat units. Table 1 gives the formulations and the vulcanization conditions for the polybutadienes; the SBR
TABLE 1 Formulation (Weight Per Weight of Polymer) and Cure of the Rubbers Used in This Study

\begin{tabular}{|c|c|c|c|}
\hline 1,4-PB & 100 & - & - \\
\hline 1,2-PB & - & 100 & - \\
\hline SBR & - & - & 100 \\
\hline $\mathrm{ZnO}$ & 3 & 2.5 & - \\
\hline Stearic acid & 2 & 2 & - \\
\hline Sulfur & 1 & 1.5 & - \\
\hline $\begin{array}{l}\text { n-Cyclohexyl-2-benzothiazolesulfenamide } \\
\text { (Santocure TBBS) }\end{array}$ & 1.2 & - & - \\
\hline $\begin{array}{l}\text { n-tert-Butyl-2-benzothiazolesulfenamide } \\
\text { (Santocure CBS) }\end{array}$ & - & 1.5 & - \\
\hline $\begin{array}{l}n \text {-(1,3-dimethylbutyl)- } n^{\prime} \text {-phenyl- } \\
\quad p \text {-phenylenediamine (Santoflex 13) }\end{array}$ & - & 1.5 & - \\
\hline \multirow[t]{2}{*}{ Cure/molding } & $160^{\circ} \mathrm{C}$ & $165^{\circ} \mathrm{C}$ & $80^{\circ} \mathrm{C}$ \\
\hline & $30 \mathrm{~min}$ & $20 \mathrm{~min}$ & n $20 \mathrm{mir}$ \\
\hline
\end{tabular}

retained its shape sufficiently that there was no need to crosslink it. Table 2 lists the glass transition temperatures of the three rubbers as measured by differential scanning calorimetry (DSC).

High rate tensile measurements (either ASTM 4482 or ASTM 1822L; samples cut from $\sim 1.5$-mm thick sheets) were made at ambient onditions using a drop-weight apparatus described elsewhere. ${ }^{37}$ The strains were determined from video images of fiducial marks recorded with a Vision Research Phantom 7 camera. At the higher strain rates, these stresses required a correction for inertial effects. ${ }^{37}$ Slower rate tensile measurements $\left(10^{-4}\right.$ to $\left.0.1 \mathrm{~s}^{-1}\right)$ used an Instron $5500 \mathrm{R}$ with an optical extensometer. Dynamic shear experiments were carried out on cylindrical samples (28-mm diameter $\times 2$-mm thick) using a Bohlin VOR rheometer. Adhesion was achieved by painting a 5\% solution of the particular polymer onto the plates, with application of a $14.7 \mathrm{~N}$ normal force at room temperature for $30 \mathrm{~min}$, following evaporation of the solvent. The shear strain for these measurements was typically $2 \%$.

\section{RESULTS}

In Figure 1, the 1,4-PB transient engineering stress and strain curves over 5.8 decades of strain rate show a monotonic increase in the slope (i.e., modulus) with rate. Measurements for the 1,2-PB (Fig. 2) at lower strain rates $(\dot{\varepsilon}<17$ $\left.\mathrm{s}^{-1}\right)$ are similar, but at higher strain rates $\left(\dot{\varepsilon} \geq 17 \mathrm{~s}^{-1}\right)$ the curves show an upward curvature at large strain $(\varepsilon>2)$. Also shown in Figure 2 is the variation of the engineering strain rate with strain, which is representative of all these measurements. At rates $>300 \mathrm{~s}^{-1}$, a constant deformation rate is not achieved until strains are around unity because of instrument inertia. Subtle transient tensile waves (speed ca. $50 \mathrm{~m} / \mathrm{s}$ ) were observed at the beginning of loading $(\varepsilon<$ 0.1 ), similar to effects described elsewhere. ${ }^{38,39}$ The strain 
TABLE 2 Glass Transition Temperatures and Fitted Parameters to eq 5 (WLF Equation)

\begin{tabular}{llll}
\hline & $1,4-\mathrm{PB}$ & $1,2-\mathrm{PB}$ & SBR \\
$T_{\mathrm{g}}(\mathrm{DSC})$ & $-93.0^{\circ} \mathrm{C}$ & $0.5{ }^{\circ} \mathrm{C}$ & $4.1^{\circ} \mathrm{C}$ \\
$T_{\mathrm{g}}\left(G^{\prime \prime}\right)$ & $-100.0^{\circ} \mathrm{C}$ & $-12.5^{\circ} \mathrm{C}$ & $2.5^{\circ} \mathrm{C}$ \\
$C_{1}$ & 2.468 & 5.423 & 7.436 \\
$C_{2}\left({ }^{\circ} \mathrm{C}\right)$ & 147.76 & 58.52 & 45.76 \\
$T_{0}\left({ }^{\circ} \mathrm{C}\right)$ & 25 & 25 & 25
\end{tabular}

rate results reported here were determined at higher strains and do not include these transient effects.

The behavior of SBR (Fig. 3) is different. At lower strain rates, the curves are similar to those at higher strain rates in Figure 2 , displaying a characteristic S-shape. However, at $\dot{\varepsilon}=10 \mathrm{~s}^{-1}$ the behavior changes. At $\varepsilon<0.2$ the stress increases markedly, then beyond $\varepsilon \sim 2$ this upturn is absent, and the failure strain is lower. At deformation rates greater than $100 \mathrm{~s}^{-1}$, the stress exhibits a maximum at $\varepsilon \sim 0.5$, followed by "strain softening." This is characteristic of yielding, rather than the anelasticity expected for rubbery materials. Video images revealed subtle necking, as expected for glassy polymers. ${ }^{40}$ There was no necking for strain rates below $100 \mathrm{~s}^{-1}$.

To compare the high strain response to the linear viscoelastic behavior, isothermal loss tangents were measured as a function of frequency $f$, then superimposed to form a master curve. The loss tangent master curves and complex shear modulus are shown in Figures 4-6 for 1,4-PB $(T=-98$ to $\left.25{ }^{\circ} \mathrm{C}\right), 1,2-\mathrm{PB}\left(-11\right.$ to $\left.25^{\circ} \mathrm{C}\right)$, and SBR $\left(7-49{ }^{\circ} \mathrm{C}\right)$, respectively. The insets of these figures display the temperaturefrequency shift factors $a_{\mathrm{T}}$, along with fits to the WilliamsLandel-Ferry (WLF) equation ${ }^{26}$

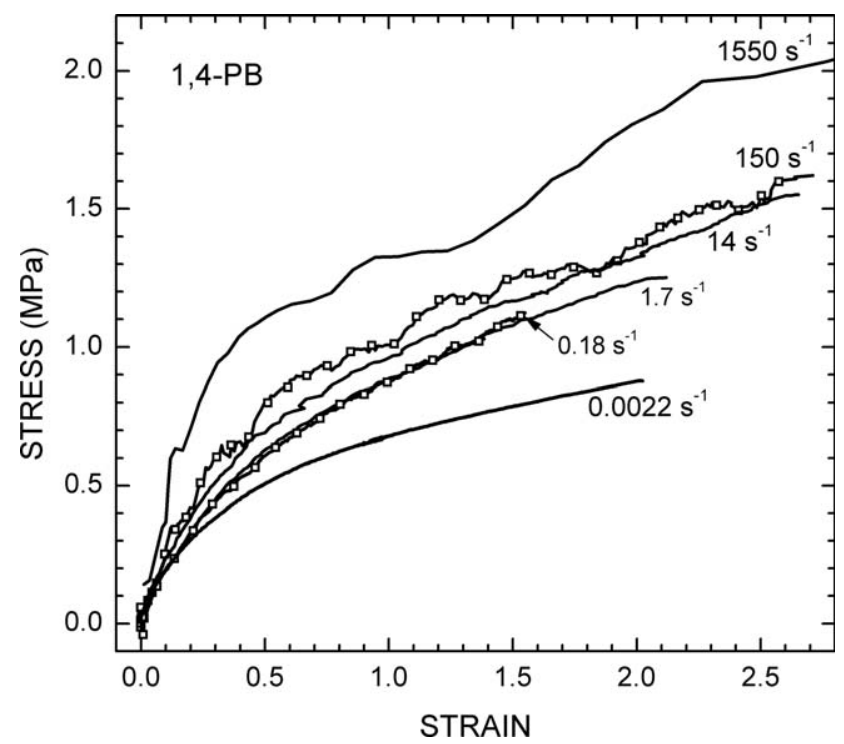

FIGURE 1 Tensile engineering stress-strain behavior of 1,4-PB, at the indicated nominal strain rates. Rates were determined by fitting a straight line to a strain versus time plot.

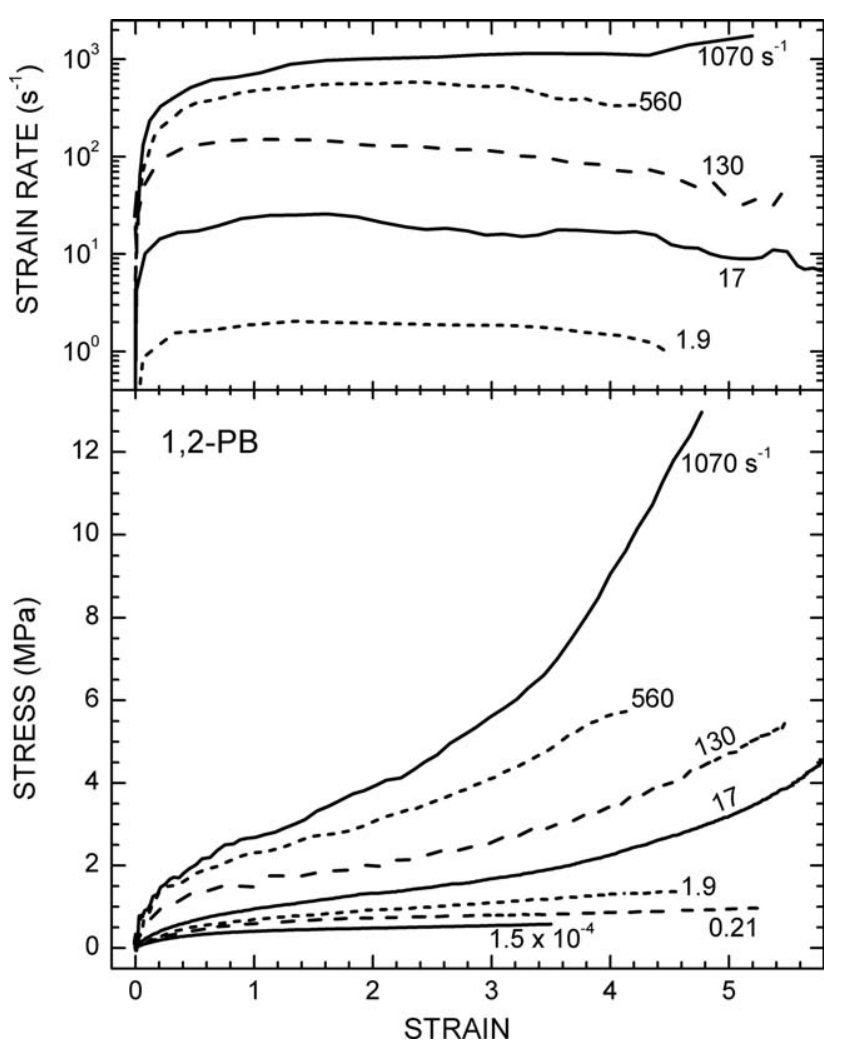

FIGURE 2 Bottom: Tensile stress-strain behavior of 1,2-PB, at the indicated nominal strain rates. Top: Strain rate as a function of strain. At the highest rates, the nominal rate was achieved at an approximate strain of unity.

$$
\log a_{\mathrm{T}}=\frac{-C_{1}\left(T-T_{0}\right)}{C_{2}+T-T_{0}}
$$

where $C_{1}$ and $C_{2}$ are constants, and $T_{0}$ is the reference temperature. Table 2 lists the WLF parameters and, for

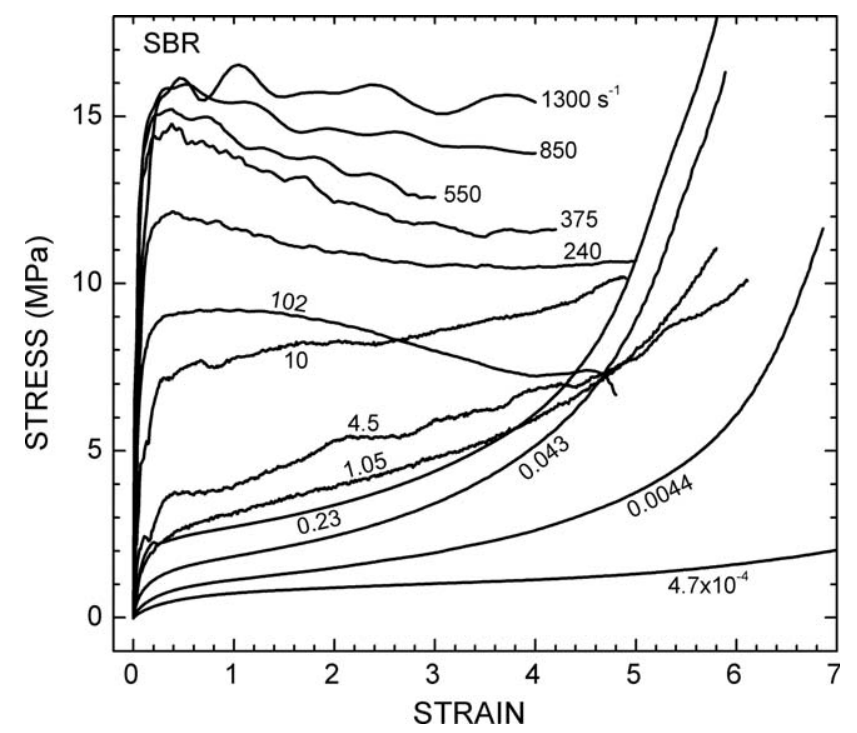

FIGURE 3 Tensile stress-strain behavior of SBR, at the indicated nominal strain rates.

JOURNAL OF POLYMER SCIENCE PART B: POLYMER PHYSICS 2011, 49, 1195-1202 


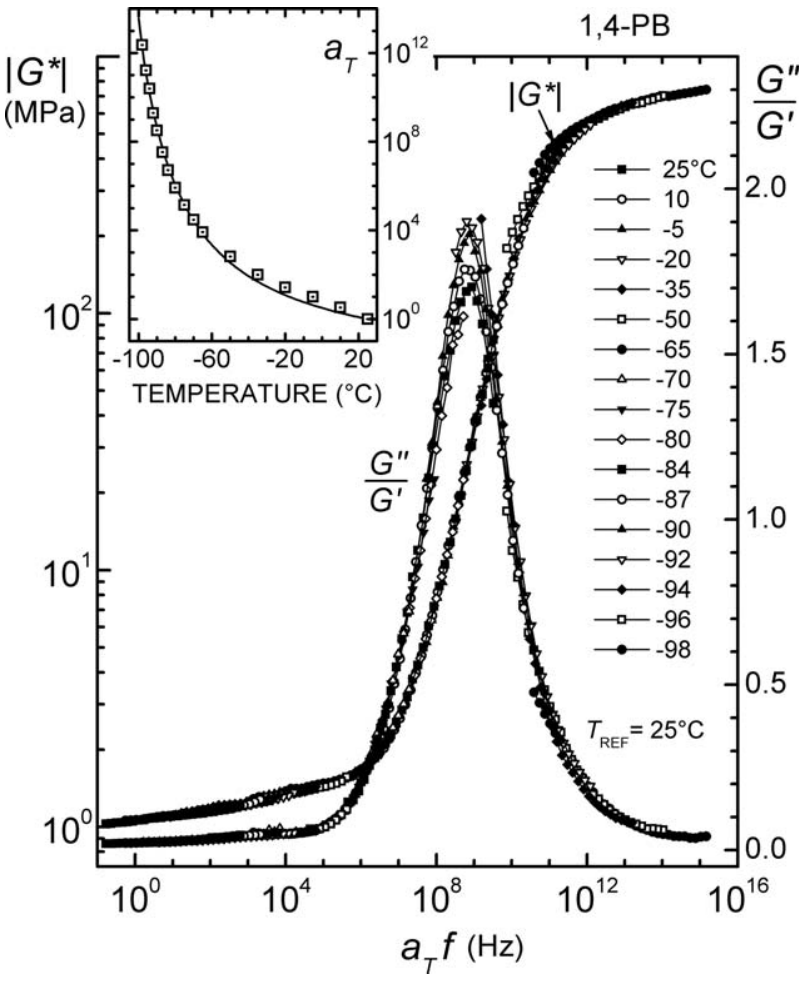

FIGURE 4 Master curves for 1,4-PB, formed by shifting the loss tangent (measured frequency range $0.017-100 \mathrm{~Hz}$ ) and the corresponding complex shear modulus magnitude. A breakdown in time-temperature superposition is evident in the peak of the loss tangent. Inset: Time-temperature shift factors ( $\log a_{T}$ ) for the corresponding loss tangent master curve. The lines are fits to the WLF equation (eq 5); the fit parameters are listed in Table 2.

comparison to the DSC values, the $T_{\mathrm{g}}$ defined as the temperature at which the inverse of the $G^{\prime \prime}$ peak frequency $\left(=\left[2 \pi \omega_{\max }\right]^{-1}\right)$ equals $100 \mathrm{s.}^{6}$

All the loss tangent peaks in Figures 4-6 show deviations from time-temperature superpositioning. This is a wellknown effect of the increasing contribution of the segmental dynamics, as temperature is decreased. ${ }^{36,41-47}$ The segmental modes have a stronger temperature dependence than the chain modes, and in the transition zone both influence the response. Nevertheless, the $a_{\mathrm{T}}$ are valid for superposition of the rubbery response up through the onset of this glass transition.

\section{DISCUSSION}

The differences in the behavior of the rubbers (Figs. 1-3) are a straightforward consequence of their different glass transition temperatures. For 1,4-PB, the transient measurements fall in the rubbery plateau region of the viscoelastic spectrum; therefore, the stress is only weakly sensitive to strain rate. For example, at $\varepsilon \sim 1$, a 10 -fold increase in rate increases the stress by just 30\%. For 1,2-PB measurements at the highest strain rates, the segmental modes contribute to the response (note the upturn in the curves at $\dot{\varepsilon}>10 \mathrm{~s}^{-1}$ in Fig. 2), and hence the strain rate sensitivity is larger than for the 1,4-PB; a 10-fold change in rate increases the stress by $80 \%$. Finally, for the SBR, a rate-induced glass transition occurs at about $\dot{\varepsilon}=102 \mathrm{~s}^{-1}$, resulting in a qualitative change in the mechanical behavior with strain rate.

The differences in sensitivity to strain rate of these polymers is a direct consequence of their different glass transition temperatures. Near $T_{\mathrm{g}}$, changes in strain rate or frequency produce comparatively larger changes in behavior. This is also apparent in the nonlinear variation of the $a_{\mathrm{T}}$ with temperature. The insets in Figures 4-6 show the shift factors for the polymers at the same reference temperature $25^{\circ} \mathrm{C}$. The slope of the shift factors at the reference temperature is given by

$$
\left.\frac{\mathrm{d}\left(\log a_{\mathrm{T}}\right)}{\mathrm{d} T}\right|_{T=T_{0}}=-\frac{C_{1}}{C_{2}} .
$$

The values are $0.0167,0.0926$, and 0.163 decades $/{ }^{\circ} \mathrm{C}$ for 1,4-PB, 1,2-PB, and SBR, respectively; hence, SBR is 10 times more sensitive to strain rate than is the $1,4-\mathrm{PB}$.

Separability of the rate and strain responses is demonstrated for 1,4-PB in Figure 7. The curves were displaced along the logarithmic ordinate to match the $\dot{\varepsilon}=1.7 \mathrm{~s}^{-1}$ data; the

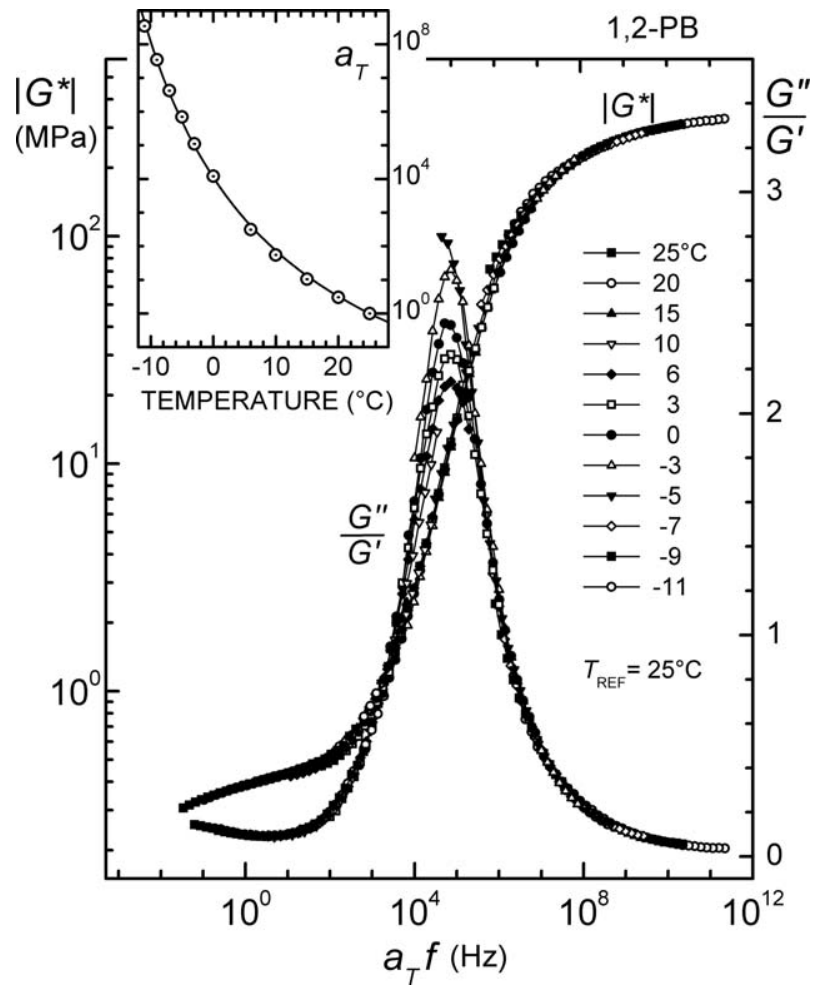

FIGURE 5 Master curve of the loss tangent for 1,2-PB (measured frequency range $10^{-3}$ to $10^{2} \mathrm{~Hz}$ ) and the corresponding complex shear modulus magnitude. Breakdown of time-temperature superposition is evident in the peak of the loss tangent. Inset: shift factors ( $\log a_{T}$ ) for the loss tangent master curve; the line is a fit to the WLF equation (eq 4), and the fit parameters listed in Table 2. 


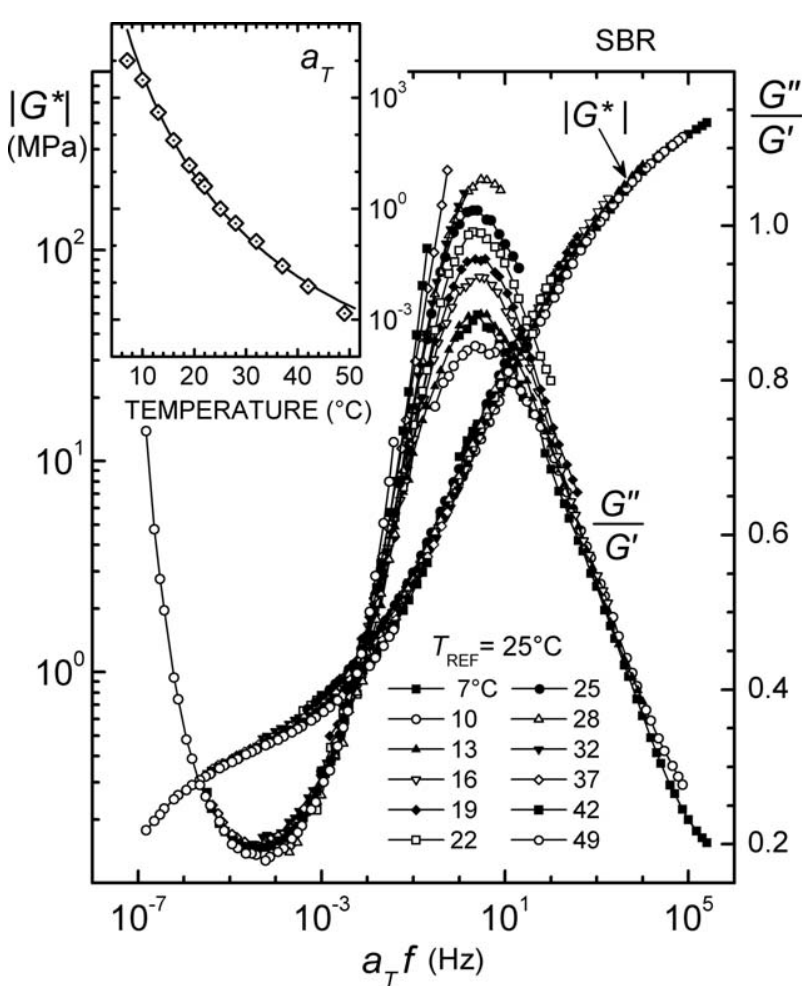

FIGURE 6 Master curve of the loss tangent for SBR (measured frequency range $10^{-4}$ to $75 \mathrm{~Hz}$ ) and the corresponding complex shear modulus magnitude. Breakdown of time-temperature superposition is evident in the peak of the loss tangent. Inset: shift factors $\left(\log a_{\mathrm{T}}\right)$ for the loss tangent master curve; the line is a fit to the WLF equation (eq 4), and the fit parameters listed in Table 2.

resulting master curve is within the scatter, except at the lowest rate. This demonstrates that the strain and rate responses are additive, conforming to eq 3 . At the lowest

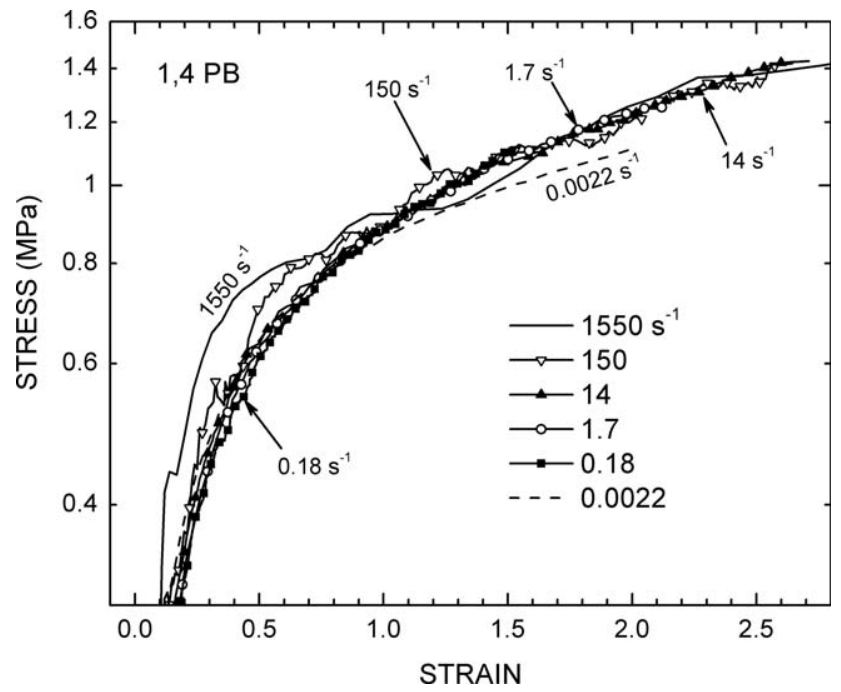

FIGURE 7 Master stress-strain curve of 1,4-PB, by vertical shifting of the curves in Figure 1 along the logarithmic ordinate. The independence from strain rate demonstrates that the time and strain response are separable. rate $\left(0.0022 \mathrm{~s}^{-1}\right)$, the stress falls below the other data for strains above 1.2. This is ascribed to the mechanically labile polysulfidic crosslinks, the dissociation of which is slow ${ }^{48-50}$ and therefore, becomes significant herein only at the lowest strain rate. For the 1,2-PB and SBR, the $\sigma-\varepsilon$ data could not be superposed, as is evident from changes in the shapes of the curves with strain rate, in Figures 2 and 3.

Figure 8 compares the linear dynamic and the nonlinear transient responses for 1,4-PB. Because the stress-strain curves include both dissipated and stored mechanical energy, the appropriate viscoelastic variable for comparison to the transient data is the complex shear modulus $\left|G^{*}\right| C=\left[\left(G^{\prime}\right)^{2}+\right.$ $\left.\left.\left(G^{\prime \prime}\right)^{2}\right]^{1 / 2}\right)$. On the ordinate, $\left|G^{*}\right|$ was converted to Young's modulus by $E=3\left|G^{*}\right|$ and plotted as tensile stress at $\varepsilon=$ 0.5 (arbitrarily selected to match the vertical location of the stress-strain data). On the abscissa, the oscillation frequency $\omega$ was converted to uniaxial strain rate using $\dot{\varepsilon}=2 \pi f$. For the transient data, the increase in $\log$ (stress) with $\log$ (strain rate) is approximately linear, with the least-squares fit indicated in Figure 8 by the dashed lines. The inset displays the slopes of these fitted power laws as a function of strain; they are constant within the scatter, $0.048 \pm 0.011 \mathrm{MPa}$ s.

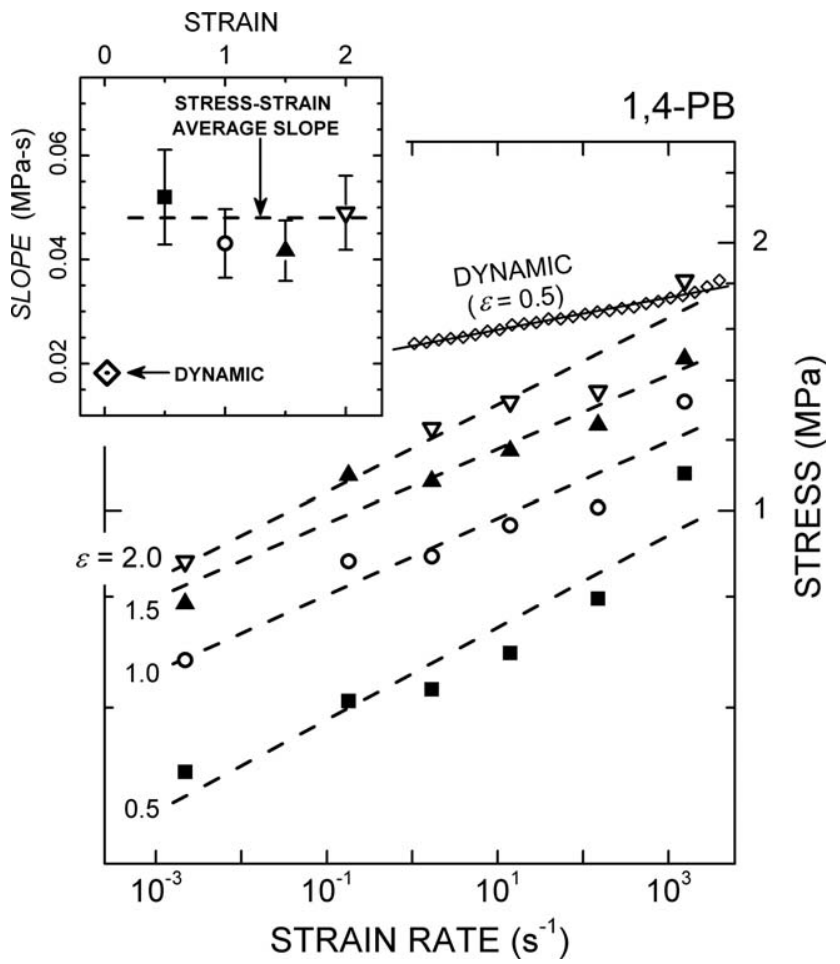

FIGURE 8 Sensitivity of stress to strain rate, at the indicated strains for 1,4-PB; note the log-log axis. The dashed straight lines are a least-square fit. For comparison, the dynamic modulus $E=3\left|G^{*}\right|$ from Figure 4 is also shown, plotted using unit strain and area, where the oscillation frequency $f$ in the abscissa was converted to strain rate by $\dot{\varepsilon}=2 \pi f$. Inset: the slopes of the least squares fit, with the indicated average, and the slope of the dynamic data. Error bars give the standard deviation of the fits. 


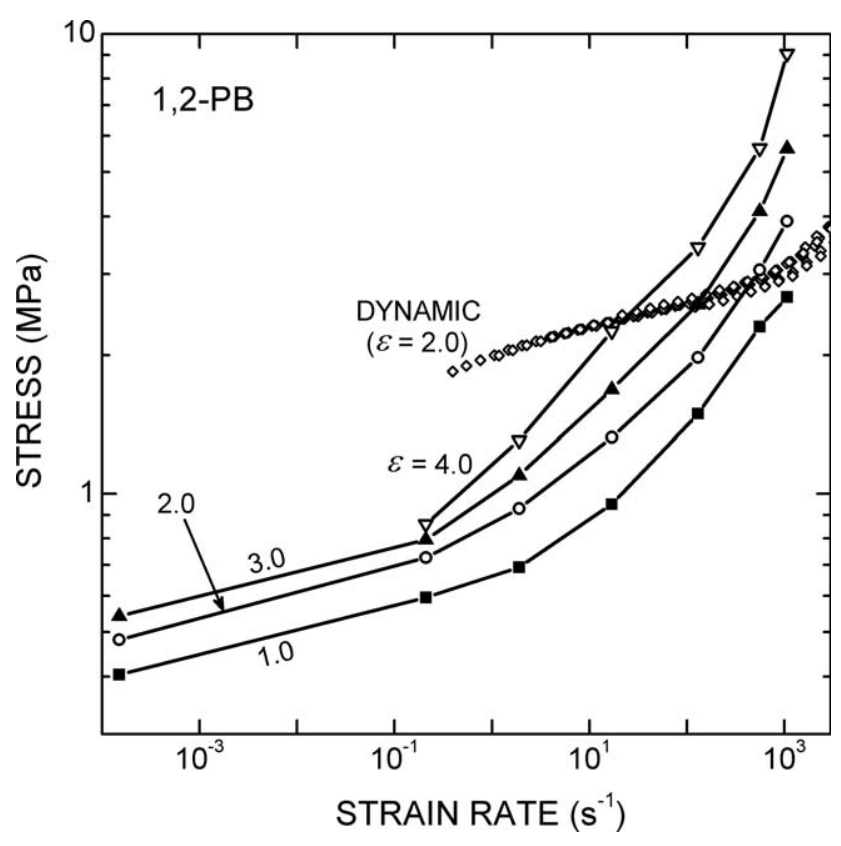

FIGURE 9 Comparison of the stress-rate curves to the viscoelastic spectrum of 1,2-PB. The stress at the indicated strains is from Figure 2. The curve for the dynamic modulus is from Figure 5 , which is converted by $E=3\left|G^{*}\right|$, plotted using $\varepsilon=2$, and with the strain rate converted from frequency by $\dot{\varepsilon}=2 \pi f$.

The slope for the dynamic data, plotted on the abscissa at $2 \%$ strain and fit only to the $25{ }^{\circ} \mathrm{C}$ measurements, is 0.0182 \pm 0.0004 MPa.s, which is 0.38 times that of the transient data slope. This difference between the measurements is greater than the scatter. Thus, dynamic measurements do not predict the rate dependence of the nonlinear, transient curves, though the strain rate and stress responses are separable. There is a lack of correspondence between oscillatory frequency and transient strain rates; eq 4 fails. Note this is not due to any failure of rate-temperature superpositioning, because these data are within the rubbery regime, where the superposition principle is valid.

While the Cox-Merz rule is often approximately valid at low to moderate shear rates $\left(\dot{\gamma}<1 \mathrm{~s}^{-1}\right)$ for which chain orientation is the dominant deformation mechanism, the contribution of chain stretching at high shear rates or in extensional flow fields is expected to produce deviations. ${ }^{22,51,52}$ This may explain the failure of the Cox-Merz rule for some branched polymers, ${ }^{18,22,53}$ which can undergo strain hardening, although there are other examples of branched polymers that exhibit correspondence between dynamic and steadystate viscosities. ${ }^{23,54}$ Considering the contribution from chain stretching, the difference between the slopes of the transient tensile and linear dynamic shear data is unsurprising.

Comparison of the linear dynamic to the transient results for 1,2-PB is shown in Figure 9. The transient data are nearly parallel with upward curvature, qualitatively different from the dynamic data. Regardless, assessments of strain energy, heat buildup, and related quantities are commonly based on linear viscoelastic dynamic measurements. ${ }^{26,55,56}$ Figure 10 plots the

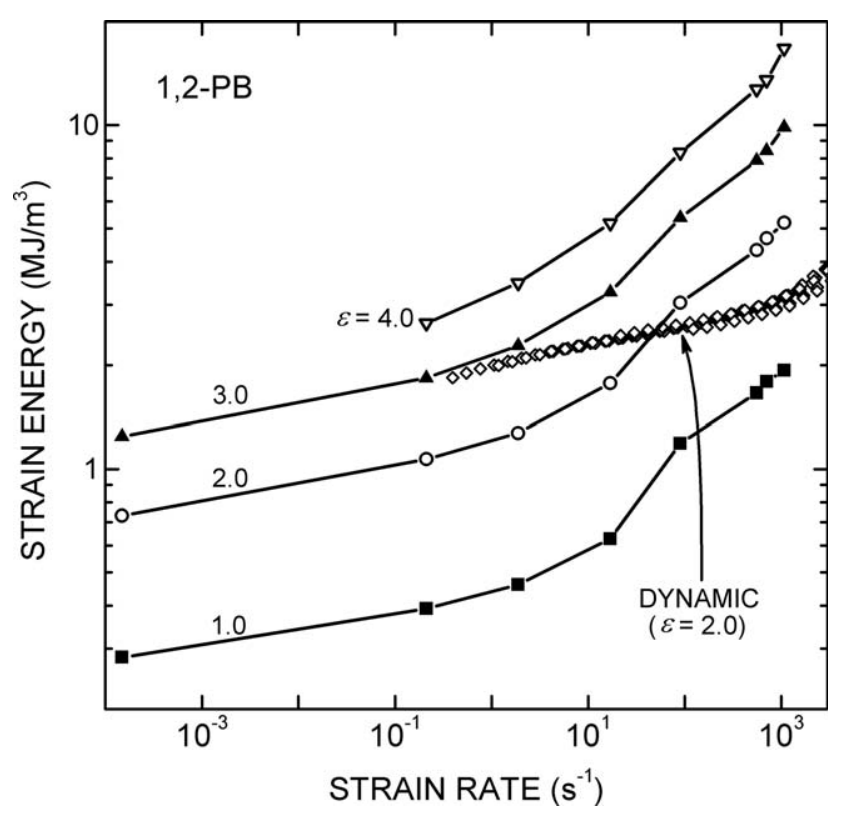

FIGURE 10 Comparison of strain energy predicted by the linear dynamic measurement, to the actual strain energy from the stress-strain measurements, for 1,2-PB, found by integrating the curves in Figure 2. The predicted strain energy was found from Figure 5 using eq 7, with the strain rate converted from frequency by $\dot{\varepsilon}=2 \pi f$.

strain energy for the 1,2-PB from integration of the $\sigma-\varepsilon$ curves (Fig. 2) for several strains. For comparison, Figure 10 shows the strain energy calculated from the dynamic data by

$$
W=\frac{1}{2} E \varepsilon^{2}=\frac{3}{2}\left|G^{*}\right| \varepsilon^{2},
$$

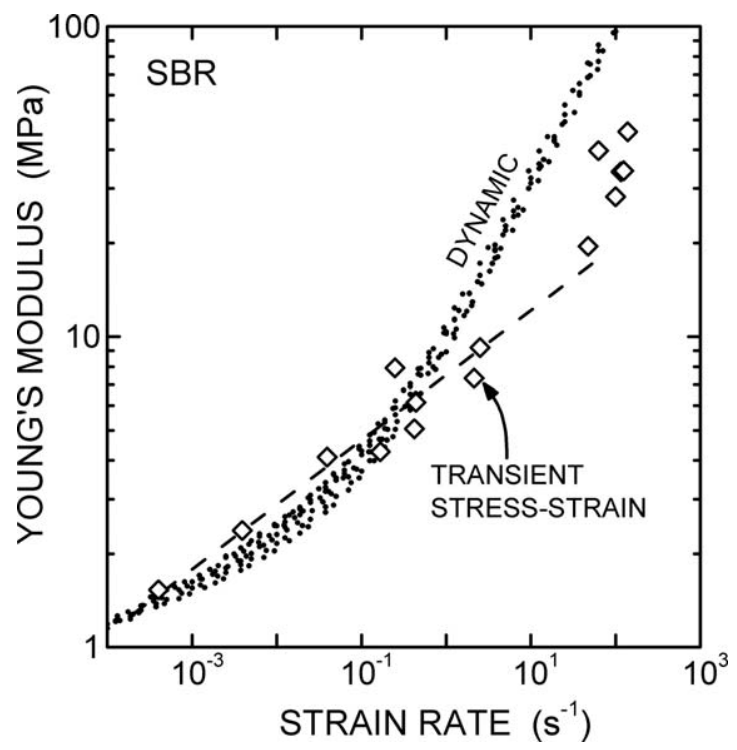

FIGURE 11 Comparison of Young's modulus from the transient stress-strain curves to the dynamic data for SBR. The transient modulus was determined by polynomial fits to Figure 3; and the dynamic modulus converted from Figure 6 (see text for details). The dashed line is a least-squares fit to the transient data when $\dot{\varepsilon} \leq 100 \mathrm{~s}^{-1}$. 
using the conversion $E=3\left|G^{*}\right|$, with $\varepsilon=2$ arbitrarily selected to match the vertical position of the stress-strain curves. The shape of the dynamic response does not match that of the transient data; thus, the strain energy calculated from linear dynamic measurements cannot be used to predict the energy for nonlinear strains. The upturn due to the onset of the transition zone occurs at a lower rate in the transient measurements than in the dynamic measurements.

In Figure 11 for the SBR, the modulus at $\varepsilon=0.1$ (before necking) was obtained by fitting a polynomial to the $\sigma-\varepsilon$ curves. This is plotted against the instantaneous strain rate and compared with the dynamic modulus (again using $E=$ $3\left|G^{*}\right|$ and $\left.\dot{\varepsilon}=2 \pi f\right)$. At the lowest rate $\left(\sim 4 \times 10^{-4} \mathrm{~s}^{-1}\right)$, the two measurements agree. However, the slope of the dynamic curve is approximately one-half that for the transient data, similar to the behavior in Figure 8 for 1,4-PB. At $\dot{\varepsilon} \sim 0.1 \mathrm{~s}^{-1}$, there is a significant increase in the slope of the dynamic data, because the segmental modes are beginning to contribute to the response, signifying the onset of the glass transition. Contrarily, the corresponding slope from the transient curve does not show such a change at this strain rate. For the transient data, the increase in slope commences around $\dot{\varepsilon} \sim 100 \mathrm{~s}^{-1}$, in agreement with the rate at which glassy deformation begins (i.e., necking and strain softening). Thus, the rate that induces a transition to the glass in the transient measurements is about three orders of magnitude greater than the corresponding transition frequency in dynamic experiments. For both 1,2-PB and 1,4-PB, at low rates within the rubbery plateau region, the dynamic and transient data at low strain agree. For 1,2-PB at high rates, there is a divergence, with the upturn in the transient measurements occurring at lower strain rates than the corresponding dynamic frequency, similar to the behavior in Figures 9 and 10.

\section{CONCLUSIONS}

For the 1,4-PB, which is within the rubbery plateau zone at room temperature, the transient stress-strain experiments conform to rate-strain separability. However, as shown in Figure 8, the variation of the modulus with strain rate is about twice its variation with dynamic (oscillation) frequency. For both the 1,2-PB and SBR, the local segmental modes begin to contribute to the transient stress-strain response at higher frequencies, with this encroachment of the transition zone convoluting the effects of strain and rate. This means that the mechanical response cannot be described using eq 3 . In addition, the effects of rate on the transient stress differ substantially from the effects of frequency on the dynamic data. Interestingly, the differences between the transient and dynamic behaviors are not consistent: For 1,2-PB, the transient upturn occurs at a frequency about three orders of magnitude lower than the upturn in the dynamic modulus; for SBR, the upturn in the transient response occurs at a frequency three order of magnitude higher than for the dynamic data. Thus, extrapolation of linear dynamic measurements to obtain predictions of stresses, strain energies, and related quantities of rubbery polymers near $T_{\mathrm{g}}$ can lead to substantial errors. The infer- ence from the Cox-Merz rule that steady-state shear deformation is approximately analogous to large transient strains is not correct.

\section{ACKNOWLEDGMENTS}

This work was supported by the Office of Naval Research, in part by Code 331, Contract Number N0001410WX21721.

\section{REFERENCES AND NOTES}

1 Bogoslovov, R; Roland, C. M; Gamache, R. M. Appl. Phys. Lett. 2007, 90, 221910.

2 Choi, I. S; Roland, C. M; Bissonnette, L. C. Rubber Chem. Technol. 1994, 67, 892-903.

3 Bekar, I; Hoo-Fatt, M. S; Padovan, J. Tire Sci. Technol. 2002, 30, 45-58.

4 Gosline, J. M. Rubber Chem. Technol. 1987, 60, 417-438.

5 Roland, C. M. Rubber Chem. Technol. 2006, 79, 429-459.

6 Roland, C. M. Macromolecules 2010, 43, 7875-7890.

7 Knauss, W. G; Sundaram, S. J. Appl. Phys. 2004, 96, 7254-7266.

8 Amirkhizi, A. V; Isaacs, J; McGee, J; Nemat-Nasser, S. Phil. Mag. 2006, 86, 5847-5866.

9 Smith, T. L. Trans. Soc. Rheol. 1962, 6, 61-80.

10 Wagner, M. H; Schaeffer, J. J. Rheol. 1993, 37, 643-661.

11 Zhao, J; Knauss, W. G; Ravichandran, G. Mech. Time-Depend. Mater. 2007, 11, 289-308.

12 Li, C; Lua, J. Mater. Lett. 2009, 63, 877-880.

13 Roland, C. M. J. Rheol. 1989, 33, 659-670.

14 Amini, M. R; Amirkhizi, A. V; Nemat-Nasser, S. Int. J. Impact Eng. 2010, 37, 90-102.

15 Sarva, S. S; Deschanel, S; Boyce, M. C; Chen, W. Polymer 2007, 48, 2208-2213.

16 Ngai, K. L; Plazek, D. J. Rubber Chem. Technol. 1995, 68, 376-434.

17 Cox, W. P; Merz, E. H. J. Polym. Sci. 1958, 28, 619-622.

18 Booij, H. C; Leblans, P; Palmen, J; Tiemersma-Thoone, G. J. Polym. Sci., Polym. Phys. Ed. 1983, 21, 1703-1711.

19 Bird, R. B; Armstrong, R. C; Hassager, O. Dynamics of Polymeric Liquids, 2nd ed.; Wiley: New York, 1987; Vol.1.

20 Venkatraman, S; Okano, M; Nixon, A. Polym. Eng. Sci. 1990, 30, 308-313.

21 Winter, H. H. Rheol. Acta 2009, 48, 241-243.

22 Ferri, D; Lomellini, P. J. Rheol. 1999, 43, 1355-1372.

23 Robertson, C. G; Roland, C. M; Puskas, J. E. J. Rheol. 2002, 46, 307-320.

24 Boltzmann, L. Pogg. Ann. Phys. 1876, Ergänzungsband 7, 624-654.

25 Yannas, I. V. J. Polym. Sci: Macromol. Rev. 1974, 9, 163-190

26 Ferry, J. D. Viscoelastic Properties of Polymers, 3rd ed.; Wiley: New York, 1980.

27 Sui, C; McKenna, G. B. J. Rheol. 2007, 51, 341-365.

28 Renardy, M. J. Non-Newton. Fluid Mech. 1997, 68, 133-135.

29 Larson, R. G. Constituitive Equations for Polymer Melts and Solutions; Buttersworth: Boston, 1988.

30 Santangelo, P. G; Roland, C. M. Rubber Chem. Technol. 1992, $65,965-972$. 
31 Quigley, C. J; Mead, J; Johnson, A. R. Rubber Chem. Technol. 1995, 68, 230-247.

32 Li, C; Lua, J. Mater. Lett. 2009, 63, 877-880.

33 Johnson, A. R; Quigley, C. J; Mead, J. L. Rubber Chem. Technol. 1994, 67, 904-917.

34 Bergström, J. S; Boyce, M. C. J. Mech. Phys. Solids 1998, 46, 931-954.

35 Qi, H. J; Boyce, M. C. Mech. Mater. 2005, 37, 817-839.

36 Roland, C. M; Ngai, K. L; Santangelo, P. G; Qiu, X. H; Ediger, M. D; Plazek, D. J. Macromolecules 2001, 34, 6159-6160.

37 Mott, P. H; Twigg, J. N; Roland, D. F; Schrader, H. S; Pathak J. A; Roland, C. M. Rev. Sci. Inst. 2007, 78, 045105.

38 Bogoslovov, R. B; Roland, C. M. J. Appl. Phys. 2007, 102, 063531. 39 Niemczura, J; Ravi-Chandar, K. J. Mech. Phys. Solids 2011, 59, 423-441.

40 Ward, I. M. Mechanical Properties of Solid Polymers, 2nd ed.; Wiley: New York, 1983; Chapter 11.

41 Plazek, D. J. J. Phys. Chem. 1995, 69, 3480-3487.

42 Plazek, D. J. Polymer J. 1980, 12, 43-53.

43 Plazek, D. J; Plazek, D. L. Macromolecules 1983, 16, 1469-1475. 44 Plazek, D. J; Chay, I. C; Ngai, K. L; Roland, C. M. Macromolecules 1995, 28, 6432-6436.
45 Santangelo, P. G; Ngai, K. L; Roland, C. M. Macromolecules 1996, 29, 3651-3653.

46 Santangelo, P. G; Roland, C. M. Macromolecules 1998, 31, 3715-3719.

47 Robertson, C. G; Rademacher, C. M. Macromolecules 2004, 37, 10009-10017.

48 Andrews, R. D; Tobolsky, A. V; Hanson, E. E. J. Appl. Phys. 1946, 17, 352-361.

49 Berry, J. P; Scanlan, J; Watson, W. F. Trans. Faraday Soc. 1956, 52, 1137-1151.

50 Scanlan, J; Watson, W. F. Trans. Faraday Soc. 1958, 54, 740-750.

51 Hua, C. C. J. Chem. Phys. 2000, 112, 8176-8186.

52 Wen, Y. H; Lin, H. C; Li, C. H; Hua, C. C. Polymer 2004, 45, 8551-8559.

53 Sui, C. P; McKenna, G. B. J. Rheol. 2007, 51, 341-365.

54 Wood-Adams, P. M. J. Rheol. 2001, 45, 203-210.

55 McCrum, N. G; Read, B. E; Williams, G. Anelastic and Dielectric Effects in Polymeric Solids; Dover: New York, 1967.

56 Gent, A. N; Scott, K. W. In Engineering with Rubber: How to Design Rubber Components, 2nd ed.; Gent, A. N., Ed.; Hanser: Munich, 2001; pp 73-97. 